\title{
Fatores associados a suplementação de ácido fólico em gestantes atendidas em uma maternidade filantrópica
}

\section{Factors associated with folic acid supplementation in pregnant women attended in a philanthropic maternity}

Gabriele Pontes da Silva 1, Adriana Sousa Rêgo 2, Andressa Pestana Brito 3, Eliziane Gomes da Costa Moura da Silva 4, Flor de Maria Araújo Mendonça 5, Gabrielle Vieira da Silva Brasil ${ }^{6}$, Allanne Pereira Araújo ${ }^{7}$, Janaina Maiana Abreu Barbosa 8.

\section{RESUMO}

O ácido fólico é uma vitamina do complexo B que possui grande importância para o crescimento e saúde do feto no fechamento do tubo neural, além de prevenir doenças e más formações fetais. A pesquisa tem como objetivo verificar os fatores associados a suplementação de ácido fólico em gestantes atendidas em uma maternidade filantrópica de São Luís - MA. Trata-se de um estudo transversal e analítico, realizado nos meses de setembro a dezembro de 2018. Utilizou-se um questionário contendo dados socioeconômicos, demográficos, estilo de vida e reprodutivos. Foram aferidas as medidas antropométricas (peso e altura). Foi realizada a análise multivariada pelo método de regressão de Poisson. Das 130 gestantes, 67,6\% referiram suplementação de ácido fólico. Durante o pré-natal, $86,15 \%$ fizeram seis ou mais consultas. Foram associados ao não uso de suplementação de ácido fólico durante a gestação está no primeiro (RP: 0,44 ; IC: $0,27-0,74$ ) e no segundo trimestre da gestação (RP: 0,30 ; IC: $0,15-0,61$ ) e ter cursado o ensino fundamental incompleto/completo (RP: 2,28 ; IC: 1,01 5,11). Faz-se necessário o planejamento de ações, enfatizando os benefícios da suplementação do ácido fólico desde o período antes da concepção, até a 14ํㅗㄴ semana gestacional.

Palavras-chave: Gestantes. Fator de risco. Ácido fólico. Suplementação.

\section{ABSTRACT}

Folic acid is a vitamin of the B complex that has great importance for the growth and health of the fetus in closing the neural tube, in addition to preventing fetal diseases and malformations. The research aimed to verify the factors associated with folic acid supplementation in pregnant women attended at a philanthropic maternity hospital in São Luís - MA. This is a cross-sectional and analytical study, carried out from September to December 2018. A questionnaire was used containing socioeconomic, demographic, lifestyle and reproductive data. Anthropometric measurements (weight and height) were measured. Multivariate analysis was performed using the Poisson regression method. Of the 130 pregnant women, $67.6 \%$ reported folic acid supplementation. During prenatal care, $86.15 \%$ had six or more consultations. They were associated with not using folic acid supplementation during pregnancy is in the first (RP: $0.44 ; \mathrm{Cl}: 0.27-0.74$ ) and in the second trimester of pregnancy (RP: $0.30 ; \mathrm{Cl}: 0,15-0.61$ ) and have completed incomplete / complete elementary school (RP: 2.28; Cl: 1.01-5.11). Action planning is necessary, emphasizing the benefits of folic acid supplementation from the period before conception until the 14th gestational week.

Keywords: Pregnant women. Risk factor. Folic acid. Supplementation.
Pós-graduanda em nutrição clínica, esportiva, estética e prescrição de fitoterápico pela Faculdade Santa Terezinha CEST.

2 Pós-doutorado em Saúde Coletiva pela Universidade Federal do Maranhão.

${ }^{3}$ Nutricionista pela Universidade CEUMA.

${ }^{4}$ Mestre em Gestão de Programas e Serviços de Saúde Universidade Ceuma.

${ }^{5}$ Doutora em Saúde Coletiva pela Universidade Federal do Maranhão.

${ }^{6}$ Nutricionista. Mestra em Saúde do Adulto e da Criança Universidade Federal

Maranhão - UFMA.

Mestre em Saúde Coletiva pela Universidade Federal do Maranhão. Docente do curso de nutrição da Faculdade Santa Terezinha - CEST

${ }^{8}$ Mestre em Saúde Coletiva pela Universidade Federal do Maranhão. Docente do curso de nutrição da Faculdade Santa Terezinha - CEST.

Email:

jana_mayana@hotmail.com 


\section{INTRODUÇÃO}

O ácido fólico é uma vitamina do complexo B que possui grande importância para o crescimento e saúde do feto no fechamento do tubo neural, além de prevenir doenças e más formações fetais (MACHIONI et al., 2013; ESPOLADOR et al., 2015). As malformações fetais são alterações que podem ser estruturais ou funcionais e cuja origem ocorre antes do nascimento. Essas modificações, dependendo do grau, acometem órgãos no início do momento embrionário que resultam em anomalias físicas ou cognitivas (AMARAL et al., 2016). Por este motivo, a suplementação dessa vitamina tem sido recomendada desde a década de 90 no Brasil. Devido à baixa aderência à ingestão de alimentos-fonte e/ou suplementação com ácido fólico durante a gestação, o governo brasileiro estabeleceu a sua fortificação nas farinhas de trigo e de milho (FUNJIMORI et al., 2013).

A Diretriz de suplementação diária de ferro e ácido fólico em gestantes afirma que a suplementação diária do ácido fólico é essencial durante o pré-natal para evitar que o bebê nasça com baixo peso, anemia materna e infecções severas durante a gravidez. Dessa forma, recomenda-se a ingestão diária de $400 \mu \mathrm{g}(0,4 \mathrm{mg})$ de ácido fólico (OMS, 2013; BRASIL, 2013a) três meses antes da concepção e até a 14ํ semana gestacional (ESPOLADOR et al., 2015).

No entanto, o organismo humano não sintetiza o ácido fólico, sendo necessário suprir as necessidades em forma de folato que são encontradas diretamente na dieta como por exemplo em fontes naturais ou através do suplemento vitamínico em comprimidos (ESPOLADOR et al., 2015). Além disso, sabe-se que o ácido fólico é considerado uma vitamina hidrossolúvel e por apresentar essa solubilidade se altera em algumas preparações, ocasionando alterações em suas concentrações e são encontrados em fontes alimentares como o feijão, vísceras, frutas cítricas e vegetais verde-escuros (ALBIERO; PEREIRA, 2016).

Dessa forma, o estado nutricional materno é um indicador de qualidade de vida tanto para a mulher quanto para o crescimento adequado do feto. A dieta no primeiro trimestre da gestação, é importante para o desenvolvimento e diferenciação dos órgãos fetais (ANDRADE et al., 2017).

Em relação aos fatores associados ao uso do ácido fólico na gestação, a literatura sugere que mulheres mais jovens, com baixa escolaridade e menor nível socioeconômico, multíparas, que relataram número de consultas de pré-natal inferior a sete e que não 
planejam a gravidez são as que utilizam em menor proporção o suplemento de ácido fólico (BARBOSA et al., 2011; LINHARES; CESAR, 2017).

A iniciativa por essa temática foi devido ao interesse em verificar os fatores associados ao uso do suplemento de ácido fólico em gestantes, devido a deficiência dessa vitamina causar um problema de saúde tanto para mãe quanto para o feto. Portanto, o objetivo deste artigo foi verificar os fatores associados a suplementação de ácido fólico em gestantes atendidas em uma maternidade filantrópica de São Luís - MA.

\section{MATERIAIS E METODOS}

Estudo transversal e analítico, realizado entre os meses de setembro e dezembro de 2018, em uma maternidade filantrópica, localizada em São Luís - Maranhão. A maternidade oferece acompanhamento para gestantes desde o pré-natal, parto e puerpério, e realiza todos os exames laboratoriais do pré-natal, preconizados pela Rede Cegonha.

Foram incluídas mulheres grávidas, em todos os trimestres, na faixa etária de 20 a 40 anos e com feto único que estavam esperando o atendimento com a equipe de saúde. Foram excluídas as gestantes adolescentes. A amostra foi do tipo não probabilística. A pesquisa foi aprovada pelo Comitê de Ética em Pesquisa (CEP) da Universidade CEUMA, conforme protocolo 2.868.719.

As gestantes responderam o questionário adaptado de Amaral et al (2016), contendo dados socioeconômicos, demográficos, estilo de vida e reprodutivos. As variáveis foram categorizadas da seguinte forma: idade em anos completos (20 a 29 e 30 a 40), renda familiar (<1 salário mínimo, 1 ou 2 salários mínimos e > 2 salários mínimos), grau de escolaridade (ensino fundamental incompleto ou completo, ensino médio incompleto ou completo, ensino superior incompleto ou completo), estado civil (vive sem companheiro e vive com companheiro), raça (negra, branca e parda), idade gestacional em semanas $\left(1^{\circ}\right.$ trimestre, $2^{\circ}$ trimestre e $3^{\circ}$ trimestre), número de consultas do pré-natal ( 0 - 6 consultas e $\geq$ 7 consultas), uso de suplementação de ácido fólico (não e sim), se trabalha no momento (não e sim), planejamento da gravidez (não e sim), uso de anticoncepcional (não e sim), números de gestações (primípara ou multípara) e dados antropométricos (peso e altura no momento da entrevista). 
O peso e a altura foram aferidos por uma balança mecânica da marca Filizolaß localizada em um piso plano. Para mensuração do peso atual e da altura foi seguido as recomendações de BRASIL (2008).

A classificação do índice de Massa Corporal (IMC) gestacional foi realizado através da curva de Atalah. O método de Atalah et al (1997) utiliza um nomograma, aplicando pontos de corte do IMC por semana gestacional para classificar a mulher a partir da $6^{\text {a }}$ semana de gestação (baixo peso: < 19,9 kg/m²; peso adequado: 20 a 24,9 kg/m²; sobrepeso: 25 a $30,0 \mathrm{~kg} / \mathrm{m}^{2}$ e obesidade: $\geq 30 \mathrm{~kg} / \mathrm{m}^{2}$ ) e até a $42^{\text {a }}$ semana (baixo peso: $<25$ $\mathrm{kg} / \mathrm{m}^{2}$; peso adequado: 25,1 a 29,2 kg/m²; sobrepeso: 29,3 a 33,2 kg/m²; e obesidade: $\geq$ $33,3 \mathrm{~kg} / \mathrm{m}^{2}$ ). E posteriormente foi categorizado em sem excesso de peso gestacional e com excesso de peso gestacional.

A análise estatística foi realizada no software Stata® 13.0 (Stata Corporation, CollegeStation, Texas, EUA). A análise descritiva das variáveis qualitativas foi descrita por frequências relativas e absolutas. Realizou-se análise multivariada, pelo método de regressão de Poisson no qual foram incluídas no modelo múltiplo todas as variáveis associadas ao evento de interesse com significância estatística de até $20 \%$. Para aceitação das associações investigadas no modelo final, foi adotado o valor de $p<0,05$. Foi considerado como variável desfecho o uso ou não da suplementação com ácido fólico e as variáveis independentes foram à situação socioeconômica, demográfica, estilo de vida, reprodutiva e antropométrica.

\section{RESULTADOS}

A amostra deste estudo constituiu-se de 130 gestantes com a faixa etária de 20 a 40 anos, sendo 66,2\% ( $n=86)$ com idade de 20 a 29 anos. Das gestantes entrevistadas, 44,6\% $(\mathrm{n}=58)$ tinham renda de 1 a 2 salários mínimos, 60,7\% ( $n=79)$ tinham ensino médio incompleto ou completo, $71,6 \% \quad(n=93)$ viviam com companheiro, 59,2\% ( $n=77)$ consideravam-se pardas e 55,4\% $(n=72)$ relataram não trabalhar no momento da entrevista (Tabela 1). 
Tabela 1. Características socioeconômica e demográficas de gestantes atendidas em uma maternidade filantrópica de São Luís- MA, 2021.

\begin{tabular}{|c|c|c|}
\hline Variável & $\mathbf{n}$ & $\%$ \\
\hline \multicolumn{3}{|l|}{ Idade } \\
\hline 20-29 anos & 86 & 66,2 \\
\hline $30-40$ anos & 44 & 33,8 \\
\hline \multicolumn{3}{|l|}{ Renda } \\
\hline < 1 salário mínimo & 51 & 39,2 \\
\hline 1 a 2 salários mínimos & 58 & 44,6 \\
\hline >2 salários mínimos & 21 & 16,2 \\
\hline \multicolumn{3}{|l|}{ Escolaridade Materna } \\
\hline $\begin{array}{l}\text { Ensino fundamental } \\
\text { Incompleto ou Completo }\end{array}$ & 13 & 10 \\
\hline $\begin{array}{l}\text { Ensino médio } \\
\text { Incompleto ou Completo }\end{array}$ & 93 & 70,7 \\
\hline $\begin{array}{l}\text { Ensino superior } \\
\text { Incompleto ou Completo }\end{array}$ & 24 & 19,3 \\
\hline \multicolumn{3}{|l|}{ Situação Conjugal } \\
\hline Vive com companheiro & 93 & 71,6 \\
\hline Vive sem companheiro & 37 & 28,4 \\
\hline \multicolumn{3}{|l|}{ Gravidez planejada } \\
\hline Não & 75 & 57,7 \\
\hline Sim & 55 & 42,3 \\
\hline \multicolumn{3}{|l|}{ Paridade } \\
\hline Um filho & 73 & 56,1 \\
\hline 2 filhos & 36 & 27,7 \\
\hline 3 ou mais filhos & 21 & 16,2 \\
\hline \multicolumn{3}{|c|}{$\begin{array}{l}\text { Suplementação de ácido } \\
\text { fólico }\end{array}$} \\
\hline Não & 42 & 32,3 \\
\hline Sim & 88 & 67,6 \\
\hline \multicolumn{3}{|c|}{$\begin{array}{l}\text { Número de consultas no } \\
\text { pré-natal }\end{array}$} \\
\hline$\geq 7$ consultas & 112 & 86,15 \\
\hline \multicolumn{3}{|l|}{$\begin{array}{l}\text { Já fez o uso de } \\
\text { anticoncepcional }\end{array}$} \\
\hline Não & 46 & 35,3 \\
\hline Sim & 84 & 64,6 \\
\hline \multicolumn{3}{|c|}{$\begin{array}{l}\text { Filho com defeito do tubo } \\
\text { neural }\end{array}$} \\
\hline \multicolumn{3}{|l|}{ Idade gestacional } \\
\hline $1^{\circ}$ trimestre & 38 & 29,2 \\
\hline $2^{\circ}$ trimestre & 45 & 34,6 \\
\hline $3^{\circ}$ trimestre & 47 & 36,2 \\
\hline
\end{tabular}




\begin{tabular}{lcc} 
Não & 72 & 55,4 \\
Sim & 58 & 44,6 \\
Raça & & 19,3 \\
Negra & 25 & 21,5 \\
Branca & 28 & 59,2 \\
Parda & 77 & $\mathbf{1 0 0}$ \\
Total & $\mathbf{1 3 0}$ & \\
\hline
\end{tabular}

Fonte: Elaborada pelos autores.

$\mathrm{Na}$ análise não ajustada foi encontrado associação significativa ao não uso de ácido fólico com as variáveis primeiro (RP: 0,23; IC: 0,11-0,47) e segundo trimestre da gestação (RP: 0,46; IC: 0,27-0,77), ter cursado o ensino fundamental incompleto/completo (RP: 1,84 ; IC: 0,74-4,59), ter realizado menos de seis consultas no pré-natal (RP: 0,15; IC: 0,02-1,04), estar desnutrida (RP: 1,57 ; IC: $0,79-3,10$ ) e ser da raça parda (RP: 2,10 ; IC: 0,90-4,92) (Tabela 2).

Tabela 2. Análise não ajustada da associação do uso do suplemento ácido fólico e varáveis socioeconômicas e reprodutivos de gestantes atendidas em uma maternidade filantrópica em São Luís - MA, 2021.

\section{Suplementação de ácido fólico}

p-valor

\begin{tabular}{|c|c|c|c|}
\hline \multirow{2}{*}{\multicolumn{4}{|c|}{$\begin{array}{l}\text { Variável } \\
\text { Idade gestacional }\end{array}$}} \\
\hline & & & \\
\hline $3^{\circ}$ trimestre & 1 & - & 1 \\
\hline $2^{\circ}$ trimestre & 0,46 & $(0,27-0,77)$ & 0,003 \\
\hline $1^{\circ}$ trimestre & 0,23 & $(0,11-0,47)$ & 0,001 \\
\hline \multicolumn{4}{|l|}{ Escolaridade } \\
\hline $\begin{array}{l}\text { Ensino superior } \\
\text { incompleto/ completo }\end{array}$ & 1 & - & 1 \\
\hline $\begin{array}{l}\text { Ensino médio incompleto/ } \\
\text { completo }\end{array}$ & 1,29 & $(0,60-2,74)$ & 0,509 \\
\hline $\begin{array}{l}\text { Ensino fundamental } \\
\text { incompleto/ completo } \\
\text { Estado nutricional }\end{array}$ & 1,84 & $(0,74-4,59)$ & 0,188 \\
\hline Eutrofia & 1 & - & 1 \\
\hline Desnutrição & 1,57 & $(0,79-3,10)$ & 0,194 \\
\hline Sobrepeso & 0,98 & $(0,55-1,77)$ & 0,972 \\
\hline Obesidade & 0,69 & $(0,26-1,84)$ & 0,468 \\
\hline \multicolumn{4}{|l|}{ Número de Consultas } \\
\hline$\geq 7$ consultas & 1 & - & 1 \\
\hline $0-6$ consultas & 0,15 & $(0,02-1,04)$ & 0,055 \\
\hline \multicolumn{4}{|l|}{ Raça } \\
\hline Branca & 1 & - & 1 \\
\hline Negra & 1,79 & $(0,67-4,78)$ & 0,244 \\
\hline Parda & 2,10 & $(0,90-4,92)$ & 0,085 \\
\hline
\end{tabular}

RP: Razão de Prevalência; IC: Intervalo de confiança

Fonte: Elaborada pelos autores. 
$\mathrm{Na}$ análise ajustada, estar no primeiro (RP: 0,44; IC: 0,27-0,74) e no segundo trimestre da gestação (RP: 0,30; IC: 0,15-0,61) e ter cursado o ensino fundamental incompleto/completo (RP: 2,28; IC: 1,01-5,11) permaneceram associados ao não uso de suplementação de ácido fólico durante a gestação (Tabela 3).

Tabela 3. Análise ajustada da associação do uso do suplemento ácido fólico e varáveis socioeconômicas e reprodutivos de gestantes atendidas em uma maternidade filantrópica em São Luís - MA, 2021.

\begin{tabular}{|c|c|c|c|}
\hline & \multicolumn{2}{|c|}{ Suplementação de ácido fólico } & p-valo \\
\hline $\begin{array}{c}\text { Variável } \\
\text { Idade gestacional }\end{array}$ & & & \\
\hline $\begin{array}{l}\text { 30 trimestre } \\
2^{\circ} \text { trimestre }\end{array}$ & $\begin{array}{c}1 \\
0,44\end{array}$ & $\begin{array}{c}- \\
(0,27-0,74)\end{array}$ & $\begin{array}{c}1 \\
0,002\end{array}$ \\
\hline $1^{\circ}$ trimestre & 0,30 & $(0,15-0,61)$ & 0,001 \\
\hline \multicolumn{4}{|l|}{ Escolaridade } \\
\hline $\begin{array}{l}\text { Ensino superior } \\
\text { incompleto/ completo }\end{array}$ & 1 & & \\
\hline $\begin{array}{l}\text { Ensino médio } \\
\text { incompleto/ completo }\end{array}$ & 1,22 & $(0,65-2,29)$ & 0,531 \\
\hline $\begin{array}{l}\text { Ensino fundamental } \\
\text { incompleto/ completo } \\
\text { Estado nutricional }\end{array}$ & 2,28 & $(1,01-5,11)$ & 0,046 \\
\hline Eutrofia & 1 & & \\
\hline Desnutrição & 1,68 & $(0,83-3,41)$ & 0,148 \\
\hline Sobrepeso & 0,99 & $(0,58-1,67)$ & 0,977 \\
\hline Obesidade & 0,64 & $(0,25-1,58)$ & 0,335 \\
\hline $\begin{array}{l}\text { Número de Consultas } \\
\geq 7 \text { consultas } \\
0-6 \text { consultas }\end{array}$ & $\begin{array}{c}1 \\
0,26\end{array}$ & $(0,04-1,70)$ & $\begin{array}{c}1 \\
0,161\end{array}$ \\
\hline Raça & & (טו, דיר, (טן & \\
\hline Branca & 1 & - & 1 \\
\hline Negra & 1,94 & $(0,79-4,78)$ & 0,145 \\
\hline Parda & 1,69 & $(0,78-3,67)$ & 0,182 \\
\hline
\end{tabular}

RP: Razão de Prevalência; IC: Intervalo de confiança

Fonte: Elaborada pelos autores.

\section{DISCUSSÃO}

Embora a Organização Mundial da Saúde (OMS) e o Ministério da Saúde recomendem o uso do suplemento de ácido fólico durante a idade fértil, em estudos já realizados no Brasil foi observado um baixo percentual de suplementação. No Rio Grande - Rio Grande do Sul em pesquisa realizada com 2.685 puérperas mostrou que 54,2\% 
usaram o ácido fólico durante a gestação (LINHARES; CESAR, 2017). Em Joinville - Santa Catarina, em estudo com 316 gestantes somente 35,4\% $(n=112)$ suplementaram o ácido fólico, em algum momento da gravidez (AMARAL et al., 2016). Uma outra pesquisa realizada em Colombo - Paraná observou que das 70 gestantes avaliadas, 35,7\% referiram não uso de ácido fólico. Esses resultados possivelmente estão relacionados ao fato dos estudos avaliarem o uso da suplementação do ácido fólico somente no período recomendado para prevenir defeitos do tubo neural.

Levando em destaque a importância do consumo do ácido fólico, foi abordado no estudo de Maia, Trevisol e Galato (2014) que uma gravidez não planejada pode levar ao não uso de suplementação necessária de ácido fólico logo no início da gravidez, e assim, comprometer o crescimento do feto. Amaral et al (2016) relataram que a gravidez não planejada e o desconhecimento da importância sobre o ácido fólico durante a gestação, são os fatores fundamentais para o não uso do suplemento.

$\mathrm{Na}$ presente pesquisa estar no primeiro e no segundo trimestre da gestação foi associado ao não uso de suplementação. Nicarretta et al (2015) realizaram entrevistas com gestantes que estavam fazendo o uso de suplemento atendidas no pré-natal do Sistema Único de Saúde (SUS), no município de Vale de Taquari (RS), observaram que 44\% das gestantes se encontravam no terceiro trimestre de gestação, acredita-se que este percentual de gestantes que estavam fazendo a suplementação de ácido fólico se deva ao aumento do número de consultas realizadas até o terceiro trimestre e dessa forma, a indicação da suplementação por parte dos profissionais de saúde. Por outro lado, Amaral et al (2016) ao avaliarem o uso de suplementação com ácido fólico e fatores associados a não realização dessa prática, observaram que o início do pré-natal anterior à 11ª semana aumentou as chances de suplementação de ácido fólico.

A assistência pré-natal é um importante componente da atenção à saúde das mulheres no período gravídico-puerperal (VIELLAS et al., 2014). Observou-se na presente pesquisa elevada cobertura da assistência pré-natal em relação ao número de consultas. Tomassi et al (2017) realizaram uma pesquisa em Unidades Básicas de Saúde (UBS) registradas no cadastro nacional de estabelecimento de saúde, localizadas na zona rural e urbana do Brasil, das 50.791 gestantes incluídas na avaliação, 89,1\% fizeram seis ou mais consultas de pré-natal. Em pesquisa realizada com 768 puérperas de Sergipe, os resultados mostraram que 74,4\% $(n=570)$ compareceram a seis ou mais consultas (MENDES et al., 2020). Em investigação cujo objetivo foi analisar a assistência pré-natal 
oferecida às gestantes usuárias de serviços de saúde públicos e/ou privados, os resultados mostraram que $73,1 \%$ das gestantes compareceram as seis consultas mínimas preconizadas pelo Ministério da Saúde (VIELLAS et al., 2014). Dado semelhante ao presente estudo. Esses resultados são satisfatórios pois atendem a uma recomendação da OMS em se realizar pelo menos seis consultas durante o pré-natal. Acredita-se que quanto maior o número de consultas maior o acompanhamento e informações sobre a importância da suplementação de ácido fólico.

Em relação a escolaridade, Lima et al. (2020) em estudo realizado com 4.036 puérperas em São Luís - MA, cujo objetivo foi descrever a prevalência e fatores associados ao uso de ácido fólico e ferro em puérperas do município, observaram que a escolaridade adequada está entre os fatores determinantes ao consumo de ácido fólico durante a gestação e o consumo é maior entre as puérperas de maior nível de escolaridade. Linhares e Cesar (2017), ao investigarem os fatores associados ao uso do suplemento de ácido fólico na gestação, apontaram que o uso de suplementação de ácido fólico foi o dobro entre as puérperas que apresentavam 12 anos ou mais de estudo comparado com as que tinham entre 0-4 anos de estudo. Amaral et al (2016) também observaram que concluir o ensino superior aumentou em 3,5 vezes a probabilidade de suplementação com folato (OR: 3,5; IC 95\%: 1,1-11,1). Segundo Marqui (2019) gestantes com maior escolaridade possuem maior acesso a informações e, consequentemente, maior conhecimento do papel protetor do ácido fólico. Logo a baixa escolaridade geralmente está relacionada ao início mais tardio de pré-natal e menor frequência de consultas (BARBOSA et al., 2011).

Por se tratar de um estudo transversal, a interpretação dos resultados em relação aos fatores associados ao uso do suplemento de ácido fólico deve levar em conta que esta é uma análise com informações de uma única abordagem, representando apenas o período em que foram coletados os dados pois em uma análise com dados provenientes de estudo com delineamento transversal as associações encontradas podem ser decorrentes de causalidade.

No entanto, estes achados destacam a relevância de esclarecer à população quanto à necessidade da utilização do ácido fólico, a fim de orientar no desenvolvimento saudável da gestação e principalmente do embrião, assim como prevenir o desenvolvimento de doenças que afetam os recém-nascidos. Além disso, mostra a importância da orientação do uso do ácido fólico, uma vez que para ser efetiva, deve ser iniciada no período préconcepcional e prolongar-se até o término do primeiro trimestre da gestação. É necessário 
que os profissionais de saúde, em especial da atenção básica, estejam atentos as mulheres que estão nos dois primeiros trimestres e com menor nível de escolaridade, já que estas são as que menos utilizam o suplemento de ácido fólico durante a gestação.

\section{CONSIDERAÇÕES FINAIS}

O uso do suplemento do ácido fólico foi apontado por mais da metade das gestantes, contudo um percentual significativo das mulheres entrevistadas não fez uso do suplemento do ácido fólico, apontando a necessidade de medidas adequadas para o aumento da utilização deste suplemento. O não consumo do suplemento de ácido fólico durante a gestação foi associado a estar no primeiro e no segundo trimestre da gestação e ao nível de escolaridade ensino fundamental incompleto/completo.

\section{REFERENCIAS}

ALBIERO, Lívia Grandini; PEREIRA, Renata Junqueira. Óbitos infantis em Palmas -TO e a sua relação com o uso de ácido fólico na gestação. Desafios - Revista Interdisciplinar Da Universidade Federal Do Tocantins, v. 3, n. 2, p. 123-131, 2016.

AMARAL, Augusto Radünz et al. Fatores associados ao uso de suplemento de ácido fólico em gestantes na cidade de Joinville, SC. Arq Catarin Med. v. 45, n. 3, p. 71-83, 2016.

ANDRADE, José Francisco Alves et al. Perfil nutricional de mães de prematuros e avaliação de diferentes fatores de risco e carências nutricionais relacionadas ao parto prematuro revisão sistemática. Para Res Med J., v.1, n.4, p. e41, 2017.

ATALAH, Samur Eduardo et al. Propuesta de un nuevo estándar de evaluación nutricional en embarazadas. Rev Med Chil. v.125, n.12, p.1429-36, 1997.

BARBOSA, Lorena et al. Fatores associados ao uso de suplemento de ácido fólico durante a gestação. Rev Bras Ginecol Obstet, Minas Gerais, v. 33, n. 9, p. 246-251, 2011.

BRASIL. Conselho Federal de Medicina. Saúde da mulher e da criança: CFM recomenda o uso de ácido fólico para gestantes. 2013a. Disponível em: https://portal.cfm.org.br/index.php?option=com content\&view=article\&id=24374:saud e-da-mulher-e-da-crianca-cfm-recomenda-o-uso-de-acido-folico-para-gestantes\&catid=3

BRASIL. Ministério da Saúde (MS). Programa Nacional de Suplementação de Ferro: manual de condutas gerais. Brasília: MS; 2013b. 
BRASIL. Ministério da Saúde. Protocolos do Sistema de Vigilância alimentar e nutricional SISVAN. Brasília: Ministério da Saúde, 2008.

ESPOLADOR, Gabriela Martins et al. Identificação dos fatores associados ao uso da suplementação do ácido fólico na gestação. Rev Enferm Cent, v. 5, n. 2, p. 1552-1561, 2015.

FUJIMORI, Elizabeth et al. Prevalência e distribuição espacial de defeitos do tubo neural no Estado de São Paulo, Brasil, antes e após a fortificação de farinhas com ácido fólico. Cad. Saúde Pública, Rio de Janeiro, v. 29, n. 1, p. 145-154, 2013.

LIMA, Renata Monteiro et al. Prevalência e fatores associados ao uso de ácido fólico e ferro em gestantes da coorte BRISA. Rev Bras Saude Mater Infant, v. 20, n. 3, p. 799-807, 2020.

LINHARES, Angélica Ozório; CESAR, Juraci Almeida. Suplementação com ácido fólico em gestantes não extremas no Sul do Brasil: prevalência e pais associados. Ciência. saúde coletiva, Rio de Janeiro, v. 22, n. 2, p. 535-542, 2017.

LUNARDI-MAIA, Tânia; SCHUELTER-TREVISOL, Fabiana; GALATO, Dayani. Uso de medicamentos no primeiro trimestre de gravidez: avaliação da segurança dos medicamentos e uso de ácido fólico e sulfato ferroso. Rev Bras Ginecol Obstet, Rio de Janeiro, v. 36, n. 12, p. 541-547, 2014.

MARCHIONI, Dirce Maria Lobo et al. Ingestão de folato nos períodos pré e pós-fortificação mandatória: estudo de base populacional em São Paulo, Brasil. Cad. Saúde Pública, Rio de Janeiro, v. 29, n.10, p. 2083-2092, 2013.

MARQUI, Alessandra Bernadete Trovó. Ácido fólico, prevenção de defeitos do tubo neural e fatores associados: uma reflexão. Temas em Educ e Saúde, Araraquara, v. 15, n. 2, p. 186-193, 2019.

MENDES, Rosemar Barbosa et al. Avaliação da qualidade do pré-natal a partir das recomendações do Programa de Humanização no Pré-natal e Nascimento. Ciênc. saúde coletiva, Rio de Janeiro, v. 25, n. 3, p. 793-804, 2020.

MURAKAMI, Patricia Yumiko; HÖFELMANN, Doroteia Aparecida. Uso de suplementos de ácido fólico e ferro em gestantes de uma unidade de saúde do Paraná. Rev Bras Pesq Saúde, Vitória, v. 18, n. 3, p. 100-113, 2016.

NICARETTA, Fernanda Micaela Richter et al. Utilização de medicamentos por gestantes usuárias do sistema único de saúde em um município do vale do Taquari - RS. Destaques Acadêmicos, Lajeado, v. 8, n. 3, p. 7-19, 2016.

Organização Mundial da Saúde (OMS). Diretriz: suplementação diária de ferro e ácido fólico em gestantes. Genebra: OMS; 2013. 
TOMASI, Elaine et al. Qualidade da atenção pré-natal na rede básica de saúde do Brasil: indicadores e desigualdades sociais. Cad. Saúde Pública, Rio de Janeiro, v. 33, n. 3, e00195815, 2017.

VIELLAS, Elaine Fernandes et al. Assistência pré-natal no Brasil. Cad. Saúde Pública, Rio de Janeiro, v. 30, supl. 1, p. S85-S100, 2014. 